\title{
Macroscopic Angular Momentum States of Bose-Einstein Condensates in Toroidal Traps
}

\author{
M. Benakli ${ }^{1}$, S. Raghavan ${ }^{1}$, A. Smerzi ${ }^{2}$, S. Fantoni ${ }^{1,2}$, and S. R. Shenoy ${ }^{1}$ \\ 1 International Centre for Theoretical Physics, I-34100, Trieste, Italy \\ 2 International School for Advanced Studies and Istituto Nazionale de Fisica della Materia, I-34014, Trieste, Italy
}

(November 21, 2016)

\begin{abstract}
We consider a Bose-Einstein condensate (BEC) of $N$ atoms of repulsive interaction $\sim U_{0}$, in an elliptical trap, axially pierced by a Gaussian-intensity laser beam, forming an effective (quasi-2D) toroidal trap with minimum at radial distance $\rho=\rho_{p}$. The macroscopic angular momentum states $\Psi_{l}(\rho, \theta) \sim \sqrt{N} \Phi_{l}(\rho) e^{i l \theta}$ for integer $l$ spread up to $\rho \lesssim \rho_{\max } \sim\left(N U_{0}\right)^{1 / 4} \gg \rho_{p}$. The spreading lowers rotational energies, so estimated low metastability barriers can support large $l \lesssim l_{\text {max }} \sim\left(N U_{0}\right)^{1 / 4}, \lesssim$ 10 for typical parameters. The $l$-dependent density profile $\left|\Phi_{l}(\rho)\right|^{2}-\left|\Phi_{0}(\rho)\right|^{2}$ is a signature of BEC rotation. Results are insensitive to off-axis laser displacements $\rho_{0}$, for $\rho_{0} / \rho_{\max } \ll 1$.
\end{abstract}

PACS: 03.75Fi, 05.30.Jp, 32.80.Pj

Bose-Einstein condensates (BEC) of atoms in magnetic traps have been the focus of intense recent activity [1] 12 . Coherence of the BEC wavefunction was demonstrated by formation of atomic interference fringes, on switchoff of weakly-coupled traps [2]. Non-destructive tests of phase-coherence, through the Josephson-like effects have been proposed [6 9], including novel self-trapping phenomena [月]. trapped BEC would be of much interest.

In bulk superfluids/superconductors, equilibrium quantized vortices 113, 14, provided classic evidence of phase coherence. In multiply-connected axial-hole geometries (e.g. thick superconducting cylinders [15] or superfluids in narrow rings), superflows with wavefunction $\Phi_{l}(\theta)=|\Phi| e^{i l \theta}$, and $|\Phi|$ uniform [16 18] maintain integer$l$ metastable quantized rotational states. BEC $l \neq 0$ states in a He II like uniform-density limit have been considered [17, 18, with the BEC rigidly restricted to flat-potential regions by (narrow-ring) rigid walls. However, one needs to go beyond this 'square-well container' limit to capture the characteristic $N$-dependent spread of BEC in a polynomial trap. The Gross-Pitaevskii equation (GPE) 19 for a non-uniform BEC includes both (repulsive) atomic collisions that spread BEC, and trap potentials, that confine it. GPE vortex solutions centered in (simplyconnected) harmonic traps have been found 10,11, but could be unstable to outward vortex displacements [12].

The investigation of $N$-atom non-uniform metastable

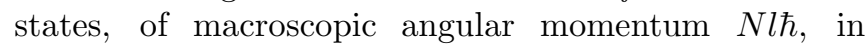
multiply-connected toroidal traps are thus important as possible signatures of BEC phase coherence. Central theoretical issues include the existence of such GPE states; the mixing of $l$-states by nonlinear GPE terms; the effects of off-center (azimuthally asymmetric) displacements of the toroidal hole; the existence of metastability barriers, and the decay-channel limiting of accessible $l$-values.

In this Letter, we consider $N$ atoms in the BEC, of interatom scattering $\sim U_{0}>0$ in an elliptical trap, with an axial 'hole' (or strong potential barrier) drilled by an intense off-resonant laser beam, forming an effective toroidal trap. The $T=0 \mathrm{GPE}$ is solved for a particular quasi-2D toroidal trap, with the ThomasFermi approximation (TFA) [3] outside an effective core, and a polynomial solution inside it. The cylindricalcoordinate wavefunctions $\Psi_{l}(\rho, \theta, z) \sim \sqrt{N} \Phi_{l}(\rho) e^{i l \theta}$ are used as bases for variational states. A dimensionless expansion parameter $\delta$, that is small for strongly interacting and dense trapped BEC, appears naturally. We find that the $l=0$ state $\Phi_{l=0}(\rho)$ of energy $E_{0} \sim \delta^{-1}$ peaks at the toroidal axis, spreading out to a radial distance $\rho=$ $\rho_{\max } \sim \delta^{-1 / 2}$. The centrifugal force induces a density fall-off $\left|\Phi_{l}(\rho)\right|^{2}-\left|\Phi_{l=0}(\rho)\right|^{2} \sim l^{2} \delta^{3}\left[\ln \left(\delta^{-2}\right)-\rho_{\max }^{2} / \rho^{2}\right]$, that is a rotation signature of the $l \neq 0$ BEC states, of rotational energy $E_{l}-E_{0} \sim l^{2}(\ln \delta) \delta \sim l^{2} / \rho_{\max }^{2}$. Offaxis displacements $\sim \rho_{0}$ of the toroidal 'hole' are unimportant if $\rho_{0} / \rho_{\max } \ll 1$. The self-interaction $U_{0}|\Psi|^{2}$, rather than destroying $l$-states, provides metastability barriers $E_{B}$ against $l \rightarrow l-1$ reductions. Other decay channels, by emission of unit-vortices, or of Bogoliubov quasi-particles, have comparable thresholds, $E_{B} \sim O(1)$. Increasing the BEC spread $\rho_{\max }$ lowers the $l \neq 0$ cost, $E_{l}-E_{0} \sim l^{2} / \rho_{\text {max }}^{2}$, so $\sim O(1)$ barriers $E_{B} \gtrsim E_{l}-E_{0}$ can still maintain large $l$ values, $l \lesssim l_{\max } \sim \delta^{-1 / 2}$. These quasi-2D trap results, as well as a square-well container limit, are recovered, from a general $d$-dimensional, anharmonic-trap scaling analysis. We now outline arguments for the above conclusions.

i) GPE and l-states: The macroscopic condensate wavefunction $\Psi(\rho, \theta, z)=\sqrt{N} \Phi(\rho, \theta, z)$ obeys the GPE in cylindrical coordinates:

$$
\begin{aligned}
& {\left[-\frac{\hbar^{2}}{2 m}\left(\frac{\partial^{2}}{\partial \rho^{2}}+\frac{1}{\rho} \frac{\partial}{\partial \rho}+\frac{1}{\rho^{2}} \frac{\partial^{2}}{\partial \theta^{2}}+\frac{\partial^{2}}{\partial z^{2}}\right)+\right.} \\
& \left.V_{\text {trap }}(\rho, \theta)+V_{\text {trap }}(z)+U_{0}|\Psi|^{2}\right] \Psi=E \Psi,
\end{aligned}
$$

where $U_{0}=4 \pi \hbar^{2} a / m$ and $a(m)$ is the atomic scattering length (mass) and $E$ is the (single-particle) energy. $V_{\text {trap }}(\rho, \theta)\left(V_{\text {trap }}(z)\right)$ is harmonic with curvature $\omega_{\|}\left(\omega_{z}\right)$. However, $V_{\text {trap }}(z)$ rises sharply for $|z| \gtrsim L_{z} / 2$, as in 
Fig. 1. The interacting BEC cloud is blocked from expanding beyond $|z| \lesssim L_{z} / 2$, spreading, for $\omega_{\|}^{2} \rho_{\max }^{2} \gg$ $\omega_{z}^{2} L_{z}^{2}$, only in the $\rho$-direction with increasing $N$. The wavefunction is quasi-2D, uniform $\sim 1 / \sqrt{L_{z}}$ in the $z$ direction. An off-resonant gaussian-profile laser barrier of high intensity $V_{c}$, and narrow width $2 \sigma$, directed along the $z$-axis, yields an axially symmetric 'doughnut' trap $V_{\text {trap }}(\rho, \theta) \rightarrow V_{\text {trap }}(\rho)$, where

$$
V_{\text {trap }}(\rho)=\frac{1}{2} m \omega_{\|}^{2} \rho^{2}+V_{c} e^{-\rho^{2} / 2 \sigma^{2}} .
$$

The BEC wavefunction is, with $l=0,1,2, \ldots$,

$$
\Phi_{l}(\rho, \theta, z) \rightarrow \Phi_{l}(\rho, \theta)=\frac{\bar{\Phi}_{l}(\bar{\rho})}{\sqrt{L_{z}} r_{\|}} \frac{e^{i l \theta}}{\sqrt{2 \pi}} .
$$

We scale lengths (energies) in the $V_{c}=0$ harmonic trap length $r_{\|}=\left(\hbar / m \omega_{\|}\right)^{1 / 2}$ (energy $\left.=\hbar \omega_{\|} / 2\right)$, so $\bar{\rho} \equiv \rho / r_{\|}, \bar{\sigma}=\sigma / r_{\|}, \bar{E}_{l}=E_{l} / \frac{1}{2} \hbar \omega_{\|}, \bar{V}_{c} \equiv V_{c} / \frac{1}{2} \hbar \omega_{\|}$. Then Eq. (11), in dimensionless form, is

$$
\begin{array}{r}
{\left[-\left(\frac{d^{2}}{d \bar{\rho}^{2}}+\frac{1}{\bar{\rho}} \frac{d}{d \bar{\rho}}\right)+\frac{l^{2}}{\bar{\rho}^{2}}+\bar{V}_{\text {trap }}(\bar{\rho})+\right.} \\
\left.\bar{N}\left|\bar{\Phi}_{l}(\bar{\rho})\right|^{2}\right] \bar{\Phi}_{l}(\bar{\rho})=\bar{E}_{l} \bar{\Phi}_{l}(\bar{\rho}),
\end{array}
$$

with $\bar{N} \propto N$ defining a central dimensionless parameter,

$$
\delta \equiv \bar{N}^{-1 / 2} \equiv\left(N_{0} / N\right)^{1 / 2} ; N_{0} \equiv L_{z} / 4 a .
$$

As shown in Fig. 2, the toroidal trap potential $\bar{V}_{\text {trap }}(\bar{\rho})=$ $\bar{\rho}^{2}+\bar{V}_{c} e^{-\bar{\rho}^{2} / 2 \bar{\sigma}^{2}}$ of Eq. (4) has a minimum around the (circular) toroidal axis at $\bar{\rho}=\bar{\rho}_{p} \equiv \sqrt{2} \bar{\sigma}\left[\ln \left(\bar{V}_{c} / 2 \bar{\sigma}^{2}\right)\right]^{1 / 2}$ : $\bar{V}_{\text {trap }}(\bar{\rho}) \approx\left(\bar{\rho}_{p}^{2} / \bar{\sigma}^{2}\right)\left(\bar{\rho}-\bar{\rho}_{p}\right)^{2}+\bar{V}_{p}$, where $\bar{V}_{p}=\bar{\rho}_{p}^{2}+2 \bar{\sigma}^{2}$. A non-interacting BEC in such a minimum has a gaussian width $r_{p}=r_{\|}\left(\sigma / \rho_{p}\right)$, i.e., the 2D 'volume' occupied is $\sim r_{\|}^{2}$. The interacting BEC spreads (as seen later) to $\bar{\rho}_{\max } \sim \bar{N}^{1 / 4}$, with a volume $\rho_{\max }^{2} \gg r_{\|}^{2}$. Thus $\delta$ of Eq. (5) is essentially the BEC volume ratio, $\delta \sim r_{\|}^{2} / \rho_{\max }^{2} \ll 1$. Note that $\delta \sim 1$ for the 'square-well' container 17, 18,20], where the BEC cannot expand.

We have solved Eq. (44) numerically. In the regime $\delta \ll 1$, and for a sufficiently low-width laser-hole, $\bar{\sigma} \ll$ $\delta^{-2 / 13}$, we find analytic results can be obtained. Dropping derivatives $\sim \delta \ll 1$ in Eq. (㺼), we have, for $\bar{\rho}$ such that $\bar{\rho}_{\text {max }}>\bar{\rho}>\bar{\rho}_{c}>\bar{\rho}_{\text {min }}$, the TFA wavefunction

$$
\bar{\Phi}_{l}(\bar{\rho})=\left(\frac{\bar{E}_{0}}{\bar{N}}\right)^{1 / 2}\left[1+\left[\overline{\mathcal{E}}_{l}-l^{2} / \bar{\rho}^{2}-\bar{V}_{t r a p}(\bar{\rho})\right] / \bar{E}_{0}\right]^{1 / 2},
$$

where $\overline{\mathcal{E}}_{l} \equiv \bar{E}_{l}-\bar{E}_{0}$ is the $l \neq 0$ energy per particle above the ground state and $\bar{\Phi}_{l}\left(\bar{\rho}_{\text {max }}\right)=\bar{\Phi}_{l}\left(\bar{\rho}_{\text {min }}\right)=0$. The wavefunction is zero for $\bar{\rho}>\bar{\rho}_{\text {max }}$; it is matched for $\bar{\rho}<$ $\bar{\rho}_{c}$ to a polynomial solution $\bar{\Phi}_{l}^{<}$inside an effective 'core' $\bar{\rho}_{c} \gtrsim \bar{\rho}_{\text {min }} . \bar{\Phi}_{l}(\bar{\rho})$ peaks at $\bar{\rho}=\bar{\rho}_{\text {peak }} \approx \bar{\rho}_{p}+\left(\bar{\sigma}^{2} / \bar{\rho}_{p}^{4}\right) l^{2}$. The energies are determined by normalization,

$$
\int_{\bar{\rho}_{c}}^{\bar{\rho}_{\max }} d \bar{\rho} \bar{\rho}\left|\bar{\Phi}_{l}(\bar{\rho})\right|^{2}+\int_{0}^{\bar{\rho}_{c}} d \bar{\rho} \bar{\rho}\left|\bar{\Phi}_{l}^{<}(\bar{\rho})\right|^{2}=1 .
$$

With the first integral dominating, we have to $O\left(\delta^{2}\right)$,

$$
\bar{E}_{l}=\bar{E}_{0}+\lambda l^{2} \delta ; \bar{E}_{0}=2 \bar{N} \delta=(4 \bar{N})^{1 / 2} .
$$

Here, $\lambda \equiv \ln \left(\bar{\rho}_{\max } / \bar{\rho}_{c}\right), \bar{\rho}_{c} \sim \bar{\sigma}\left[\ln \left(\bar{V}_{c} / \bar{E}_{0}\right)\right]^{1 / 2}$, so the results are only very weakly dependent $\left(\lambda \sim \ln \bar{\rho}_{c} \sim\right.$ $\left.\ln \left[\ln \left[\bar{V}_{c} / \bar{\sigma}\right]\right]\right)$ on the toroidal core region and how it is modeled. The interaction pushes the BEC far beyond the toroidal axis, to $\bar{\rho}=\bar{\rho}_{\max }(l) \gg \bar{\rho}_{p}$, and with $\bar{\rho}_{m} \equiv \bar{\rho}_{\text {max }}(l=0)$, we have

$$
\bar{\rho}_{\text {max }}(l)=\bar{\rho}_{m}\left(1+\frac{\lambda l^{2} \delta^{2}}{4}\right) ; \bar{\rho}_{m} \simeq(4 \bar{N})^{1 / 4}=\bar{E}_{0}^{1 / 2} .
$$

The local density difference due to centrifugal effects $\sim$ $l^{2} / \rho^{2}$ in Eq. (4) is

$$
\left|\bar{\Phi}_{l}(\bar{\rho})\right|^{2}-\left|\bar{\Phi}_{l=0}(\bar{\rho})\right|^{2}=\lambda l^{2} \delta^{3}\left[1-\left(\bar{\rho}_{m}^{2} / 2 \lambda \bar{\rho}^{2}\right)\right],
$$

with $\bar{\Phi}_{l}$ crossing $\bar{\Phi}_{0}$ at $\bar{\rho} \approx \bar{\rho}_{m} / \sqrt{2 \lambda}$.

Returning to the $\bar{\rho}<\bar{\rho}_{c}$ series solution, $\bar{\Phi}_{l}^{<}(\bar{\rho})=$ $\sum_{i=0}^{2} a_{2 i} \bar{\rho}^{2 i+l}$, we find that the coefficients are $a_{0} \sim$ $a_{4} / \bar{V}_{c}^{2}, a_{2} \sim a_{4} / \bar{V}_{c}$, with $a_{4} \sim \bar{\sigma}^{3 / 2} \delta$. The TFA $\bar{\Phi}_{l}(\bar{\rho})$ falls off as $\sim\left(\bar{\rho}-\bar{\rho}_{\text {min }}\right)^{1 / 2}$, with $\bar{\rho}_{\text {min }} \simeq \sqrt{2} \bar{\sigma}\left[\ln \left(\bar{V}_{c} / \bar{E}_{0}\right)\right]^{1 / 2}$. The wavefunctions and derivatives can be matched at a $\bar{\rho}_{c} \equiv[(l+4) /(l+7 / 2)] \bar{\rho}_{\text {min }}>\bar{\rho}_{\text {min }}$, and the $\bar{\Phi}_{l}^{<}$integral in Eq. (7) is $\sim a_{4}^{2} \bar{\rho}_{c}^{10} \sim \bar{\sigma}^{13} \ln \left(\bar{V}_{c} \delta\right) \delta^{2} \ll 1$.

Figure 2 shows $\left|\bar{\Phi}_{l}(\bar{\rho})\right|^{2}$ versus $\bar{\rho}$, for $l=0$ and 10 , for parameters as given later. The analytic approximation (solid line) closely matches the (dashed line) numerical solution of Eq. (4). The centrifugal reduction and shift of the density peak ( $10 \%$ for $l=10)$, is a rotation signature, possibly detectable by phase contrast imaging [1]. The super-current density is $j \sim$ $\hbar /(i m 2 \pi \bar{\rho}) \Psi^{*} \frac{\partial}{\partial \theta} \Psi=N\left|\bar{\Phi}_{l}(\bar{\rho})\right|^{2} v_{s \theta}$, where the azimuthal velocity $v_{s \theta}=(\hbar l / 2 \pi m \rho)$, and $j$ vanishes at the origin as $j \sim \rho^{2 l+3}$. The laser hole 'pins' the azimuthal velocity of average angular momentum $\left\langle\Psi_{l}|(\hbar / i) \partial / \partial \theta| \Psi_{l}\right\rangle=N l \hbar$, suppressing displacement instabilities [12].

ii) Metastability barriers: The nonlinear term in the GPE could cause mixing of $l$-states and $l \rightarrow$ $l-1$ decays, but instead, induces barriers. A GPE trial function for full superposition is $\bar{\Psi}(\bar{\rho}, \theta) / \sqrt{N}=$ $\left[C_{l} \bar{\Phi}_{l}(\bar{\rho}, \theta)+C_{l-1} \bar{\Phi}_{l-1}(\bar{\rho}, \theta)\right]$, with variational constants, $\left|C_{l}\right|^{2}+\left|C_{l-1}\right|^{2}=1$. With an external angular velocity $\Omega \equiv \bar{\Omega} \omega_{\|} / 2$ in the hamiltonian $\hat{H} \rightarrow \hat{H}-\hbar \Omega \hat{L}$, the free energy functional $\bar{F}(\bar{\Psi})$ (whose variation is Eq. (11)) is given by,

$$
\begin{aligned}
\frac{\bar{F}}{N} & =\sum_{p=l, l-1}\left\{\bar{E}_{p}(\bar{\Omega})-I_{p, p}\left(1-\left|C_{p}\right|^{2}\right)\right\}\left|C_{p}\right|^{2} \\
& +4\left|C_{l}\right|^{2}\left|C_{l-1}\right|^{2} I_{l, l-1},
\end{aligned}
$$

where $I_{l, l^{\prime}} \equiv \frac{1}{2} \bar{N}\left\langle\left\langle\left|\bar{\Phi}_{l}(\bar{\rho})\right|^{2}\left|\bar{\Phi}_{l^{\prime}}(\bar{\rho})\right|^{2}\right\rangle\right\rangle$, with $\langle\langle\ldots\rangle\rangle \equiv$ $\int_{0}^{\infty} d \bar{\rho} \bar{\rho}^{d-1} \ldots$, and $d=2$ at present, and $\bar{E}_{l}(\bar{\Omega}) \equiv \bar{E}_{l}-l \bar{\Omega}$. 
The $l$-state $\left(C_{l}, C_{l-1}\right)=(1,0)$ is separated from the $l-1$ state $(0,1)$ by a barrier of height $\bar{F}_{B} / N \equiv \bar{E}_{B} \equiv \bar{E}-$ $\bar{E}_{l}(\bar{\Omega})$, larger than the splitting, $\Delta \bar{E}_{l}=(2 l-1) \lambda \delta$. The 'full-overlap' barrier $\bar{E}_{B}=\bar{E}_{B 1}$ is at $\left|C_{l}\right|^{2}=\left|C_{l-1}\right|^{2}=$ $\frac{1}{2}+O\left(\delta^{2}\right)$,

$$
\bar{E}_{B 1}=2 \bar{E}_{0}\left[1-\lambda\left(l^{2}+(l-1)^{2}\right) \delta^{2}\right]-\left(\Delta \bar{E}_{l}-\bar{\Omega}\right) .
$$

Thus $\bar{E}_{B 1} \sim \bar{E}_{0} \sim \bar{N}^{1 / 2}$, and the $l$-state $(1,0)$ is preserved by metastable barriers for $\overline{\mathcal{E}}_{l}=l^{2} \lambda / \sqrt{\bar{N}}<\bar{E}_{B 1}$ or $l<\bar{N}^{1 / 2}$. These barriers arise because interatomic collisions disfavor the transition-state slowing down of a minority fraction $(<1 / 2)$ of the rotating flow.

We now consider a superposition of non-uniform $l$ states, overlapping only at an interface, lowering the $l, l-$ 1 interspecies scattering. With $\nu_{l}, \nu_{l-1}$, the fractional volumes occupied, and $\nu_{0} \equiv\left(\nu_{l}+\nu_{l-1}-1\right)>0$ the overlap fraction, we take $\bar{\Phi}_{p}(\rho) \rightarrow \bar{\Phi}_{p}(\bar{\rho}) g_{p}(\bar{\rho})$ where the stepfunction $g_{l-1}(\bar{\rho})\left(g_{l}(\bar{\rho})\right)$ is unity for $\nu_{l-1} \bar{\rho}_{m}^{2}>\bar{\rho}^{2}>\bar{\rho}_{c}^{2}$ (for $\left.\bar{\rho}_{m}^{2}>\bar{\rho}^{2}>\left(1-\nu_{l}\right) \bar{\rho}_{m}^{2}\right)$ and zero otherwise. Repeating the previous argument with these wavefunctions, we find that the intra-species scattering is increased by the confinement to a reduced volume $\nu_{p} \bar{\rho}_{m}^{2}<\bar{\rho}_{m}^{2}$, so the TFA energies are raised, $\bar{E}_{p}(\nu) \simeq I_{p, p}(\nu) \simeq \bar{E}_{p}(\nu=1) / \nu_{p}$. The inter-species scattering gives $I_{l, l-1}(\nu) \simeq \nu_{0} \bar{N}^{1 / 2} / \nu_{l} \nu_{l-1}$. The lowest interface-overlap barrier $\bar{E}_{B 2}$ obtains for approximately equal volume fractions $\nu_{l}=\nu_{l-1} \gtrsim 1 / 2$, and for an annular overlap volume of radius $\bar{\rho}_{m} / \sqrt{2}$ and thickness $\sim \bar{\xi}$. Thus $\nu_{0}=\sqrt{2} \bar{\xi} \bar{\rho}_{m} ; \bar{E}_{B 2}$ is a fraction of the full-overlap barrier, $\bar{E}_{B 2} \sim\left(\sqrt{2} \bar{\xi} / \bar{\rho}_{m}\right) \bar{E}_{B 1}$. (Gradient contributions omitted in TFA would be comparable). Here $\bar{\xi} \sim 1 /\left[\bar{N}\left|\bar{\Phi}_{l}\left(\bar{\rho}_{p}\right)\right|^{2}\right]^{1 / 2} \sim 1 / \bar{E}_{0}^{1 / 2}$ is a 'healing length', so $\bar{E}_{B 2}=2 \sqrt{2} \sim O(1)$, maintaining $l$-states for $l \lesssim l_{\max 2}=(2 \sqrt{2} / \lambda)^{1 / 2} \delta^{-1 / 2}, \sim \bar{N}^{1 / 4}$. The external angular velocity induces an $l \rightarrow l-1$ transition only above critical values, $|\bar{\Omega}| \geq\left|\bar{\Omega}_{c l}\right|=\left|\left(\bar{E}_{B}-\Delta \bar{E}_{l}\right)\right|$. This $\bar{\Omega}_{c l} \sim \bar{E}_{B 2}$ estimate exceeds an $\bar{\Omega}_{c l} \sim\left|\Delta \bar{E}_{l}\right|$ estimate [4] based on the splitting alone.

Wedge-shaped $l-1$ regions, expanding azimuthally, would have $\sim\left(2 \bar{\xi} / \bar{\rho}_{m}\right) \bar{E}_{B 1}$ interface costs, and similar $l_{\max } \sim \bar{N}^{1 / 4}$ scaling. $2 \mathrm{D}$ phase-slip saddle-point solutions [18,20] would also provide such barriers.

iii) Off-center toroidal hole: In practice, the toroidal 'hole' of Fig. (1) could be drilled off the elliptical-trap axis, or the laser could fluctuate in profile and position. With a displacement $\vec{\rho}=\left(\rho_{0}, \theta\right)$ of the $\sim \rho^{2}$ elliptical trap, $V_{\text {trap }}(\rho) \rightarrow V_{\text {trap }}+\bar{\rho}^{2}-2 \bar{\rho}_{0} \bar{\rho} \cos \theta$, modifying the TFA solution of Eq. (6). Repeating the above argument, the BEC cloud becomes anisotropic, extending to $\bar{\rho}_{m}=\bar{\rho}_{0} \cos \theta+\bar{E}_{0}^{1 / 2}$, but global averages of the energy/angular momentum are unaffected, to order $\sim \bar{\rho}_{0} / \bar{\rho}_{m} \ll 1$. Although $C_{l} C_{l-1}^{3}$ terms (previously zero by $e^{i l \theta}$ orthogonality) now enter in Eq. (11), the barriers $\bar{E}_{B 1,2}$ to $l$-decay are similarly insensitive.

iv) l-decay channels: An l-state could decay through (rectangular) unit-vortex loops, with straight segments of vorticity $J_{z}=-1$ reducing core vorticity by $l \rightarrow l-1$, that pushes the $J_{z}=1$ segment outwards by a repulsive energy $\sim-(l-1) \ln \rho$. With $\left|\bar{\Phi}\left(\bar{\rho}_{c}\right)\right|^{2} \propto\left(\bar{\rho}_{c}-\bar{\rho}_{m}\right) \sim$ $\bar{\rho}_{c} \sim \bar{\sigma}$, the nucleation cost near the core is $\bar{E}_{B 3} \sim$ $\bar{\xi}^{2} \bar{N}\left|\bar{\Phi}_{l}\left(\bar{\rho}_{c}\right)\right|^{2} \sim \bar{\sigma}$. Then $\overline{\mathcal{E}}_{l}=\lambda l^{2} \delta<\bar{E}_{B 3}$ accesses this decay channel only for $l \geq l_{\max 3} \sim \sqrt{\bar{\sigma}} \bar{N}^{1 / 4} / \sqrt{\lambda}$. (As $v_{s \theta} \bar{\rho} \sim \hbar l / 2 \pi m$ is a constant, $v_{s \theta}$ should not induce a loop-expanding current-drive force [14].)

Another possible decay channel is a successive reduction $N \rightarrow N-2$ of BEC $l$-state atoms, producing surface pairs of dissipative quasiparticles [11] of Bogoliubov excitation energy $2 \bar{\Delta}$, and angular momentum $l \pm q[17,18]$, that slow down to fall into the $l=0$ ground state. A detailed analysis [16] for toroidal traps is beyond the scope of this paper; however, for $\overline{\mathcal{E}}_{l} \lesssim \bar{\Delta}$, the quasiparticle channel is not accessed for $l \lesssim \sqrt{\Delta} \bar{N}^{1 / 4}$. In other contexts [21], quasiparticle damping is small.

v) Numerical estimates: Parameters chosen are $\omega_{\|}=$ $132 \mathrm{rad} \mathrm{s}^{-1},(1 \mathrm{nK}), r_{\|}=6.3 \mu \mathrm{m}, L_{z}=25 \mu \mathrm{m}, 2 \sigma=$ $12 \mu m, a=50 \AA, V_{c}=63 n K$, and $m=2 \times 10^{-26} \mathrm{~kg}$ $(\sim$ sodium $)$. Then $\rho_{p}=17.5 \mu m, N_{0} \approx 10^{3}$, and for $N=10^{6}$, we have $\delta=1 / \sqrt{\bar{N}}=0.03$. Physical magnitudes are then $E_{0}=32 n K, \lambda=2, \mathcal{E}_{l}=0.031 l^{2} n K, \rho_{m}=$ $50 \mu m, \xi=0.8 \mu m, v_{s \theta}(\rho) \leq v_{s \theta}\left(\rho_{c}\right)=0.12 l \mathrm{~mm} \mathrm{~s}^{-1}, \Omega_{c l}=$ $30 \mathrm{~Hz}$, and $l_{\max 2} \sim 10$. Thermal excitations activated over $N E_{B}$ free energy barriers are exponentially dilute, at low temperatures.

Finally, we present a general $\bar{N}$-scaling argument. Consider a $d$-dimensional toroidal trap that, for distances $\bar{r}$ well away from the core, is $V_{\text {trap }} \sim \hbar \omega_{t} \bar{r}^{\alpha}$, with $\bar{r} \equiv r / r_{t}$ and $r_{t}=\left(\hbar / m \omega_{t}\right)^{1 / 2}$. For $\alpha=2$ the trap is harmonic; for $\alpha \rightarrow \infty$, a square-well container results, $V_{\text {trap }}=0(\infty)$ for $r<r_{t}\left(r>r_{t}\right)$. Then defining $\bar{N} \equiv\left(N U_{0} / \hbar \omega_{t}\right) r_{t}^{d}, \bar{\Phi}(\overrightarrow{\vec{r}}) \equiv$ $\Phi(\vec{r}) / r_{t}^{d / 2}, \bar{E}_{l} \equiv E_{l} / \hbar \omega_{t}$, the TFA wavefunction, $\bar{\Phi}_{l}=$ $\left(\left[\bar{E}_{l}-\bar{r}^{\alpha}-\left(l^{2} / \bar{r}^{2}\right)\right] / \bar{N}\right)^{1 / 2}$ is almost flat, $\left|\bar{\Phi}_{l}(\bar{r})\right| \simeq\left|\bar{\Phi}_{0}\right| \approx$ $\left(\bar{E}_{0} / \bar{N}\right)^{1 / 2}$, with BEC spreading until $\bar{r} \simeq \bar{r}_{m}$, when the confining potential rises to the energy, $\bar{E}_{l} \sim \bar{r}_{m}^{\alpha}$. With normalization $\left\langle\left\langle\left|\bar{\Phi}_{l}(\bar{r})\right|^{2}\right\rangle\right\rangle \simeq\left|\bar{\Phi}_{0}\right|^{2} \bar{r}_{m}^{d}=1$, we obtain $\bar{r}_{m}=\bar{N}^{1 /(\alpha+d)}$. Then $\overline{\mathcal{E}}_{l} \sim l^{2} / \bar{r}_{m}^{2}$, and the healing length is $\bar{\xi} \sim\left(\bar{N}\left|\bar{\Phi}_{0}\right|^{2}\right)^{-1 / 2} \sim \bar{N}^{-\alpha / 2(\alpha+d)}$. The integral $I_{l, l-1} \sim \bar{N}\left\langle\left\langle\left|\bar{\Phi}_{l}(\bar{r})\right|^{2}\left|\bar{\Phi}_{l-1}(\bar{r})\right|^{2}\right\rangle\right\rangle$ defines a fulloverlap metastability barrier $\bar{E}_{B 1} \sim I_{l, l-1} \sim\left(\bar{N} / \bar{r}_{m}^{d}\right)$; while for a $d-1$ dimensional $l, l-1$ interface of thickness $\bar{\xi}, \bar{F}_{B 2} / N \equiv \bar{E}_{B 2}=\left(\bar{\xi} / \bar{r}_{m}\right) \bar{E}_{B 1}$. The vortex nucleation threshold is $\bar{E}_{B 3} \sim \bar{\xi}^{2} \bar{r}_{m}^{d-2} \bar{N}|\bar{\Phi}|^{2} \sim \bar{r}_{m}^{(d-2)}$. The $l$-state energies $\overline{\mathcal{E}}_{l}$ exceed these barriers only for $l$ greater than $l_{\max 1} \sim \bar{N}^{(2+\alpha) / 2(\alpha+d)}, l_{\max 2} \sim \bar{N}^{(2+\alpha) / 4(\alpha+d)}, l_{\max 3} \sim$ $\bar{N}^{d / 2(\alpha+d)}$. For $\alpha=d=2$, we recover our results, with $l_{\max 2} \sim l_{\max 3} \sim \bar{N}^{1 / 4}$. For $\alpha \rightarrow \infty, \bar{r}_{m}=1$, i.e. a restricted $r_{m}=r_{t}$, and the familiar He II healing length $\bar{\xi} \sim\left(N U_{0}\right)^{-1 / 2}$ is recovered, with energies $\bar{E}_{l} \sim \bar{N}+l^{2}$, and barriers $\bar{E}_{B 1} \sim \bar{N}, \bar{E}_{B 2} \sim \bar{N}^{1 / 2}, \bar{E}_{B 3} \sim O(1)$. The $l$-bounds are $l_{\max 1}=\bar{N}^{1 / 2}, l_{\max 2} \sim \bar{N}^{1 / 4}, l_{\max 3} \sim 0(1)$ : narrow-ring containers [17, 18,20 would suppress vortex- 
loops and make quasi-1D phase-slips [20] the lowest decay thresholds, $F_{B 2} \sim N^{3 / 2} U_{0}^{1 / 2}$. Barriers vanish in the ideal gas limit, $U_{0} \rightarrow 0$.

Experimental preparation of $l \neq 0$ states could be through stirring normal states by a laser 'paddle-wheel' [1] and cooling below transition; or by rotating the trap [22] with $\Omega>\Omega_{c l}$, below $T_{c}$. Experimental detection might be through the $l \neq 0$ density profiles, or the Sagnac effect 18] in large azimuthal-velocity regions around the core. Another interesting setup is a double-toroidal trap, with $N_{1}=N, l_{1}=1\left(N_{2}=N, l_{2}=0\right)$ for toroid 1(2), where the toroids initially are separated by a blocking laser sheet, whose removal could result in inter-trap 'macroscopic quantum coherence' 23.

In conclusion, $l \neq 0$ macroscopic angular momentum states of condensates, with $l$-dependent density profiles, can exist in toroidal traps, maintained by atomic collisions, and robust to toroidal hole displacements, and to estimated decays, for $l$ not too large. The observation of these states would be a signature of macroscopic phase coherence of trapped Bose-Einstein condensates.

[1] M. H. Anderson, M. R. Matthews, C. E. Wieman, and E. A. Cornell, Science 269, 198 (1995); K. B. Davis, M.O. Mewes, M. R. Andrews, N. J. van Druten, D. S. Durfee, D. M. Kurn, and W. Ketterle, Phys. Rev. Lett. 75, 3969 (1995); C. C. Bradley, C. A. Sackett, J. J. Tollett, and R. G. Hulet, Phys. Rev. Lett. 75, 1687 (1995).

[2] M. R. Andrews, C. G. Townsend, H.-J. Miesner, D. S. Durfee, D. M. Kurn, and W. Ketterle, Science 275, 637 (1997).

[3] M. Edwards and K. Burnett, Phys. Rev. A 51, 1382 (1995); P. A. Ruprecht et al., Phys. Rev. A 51, 4704 (1995).

[4] G. Baym and C. J. Pethick, Phys. Rev. Lett. 76, 6 (1996).

[5] A. Griffin, Phys. Rev. A 53, 9341 (1996).

[6] J. Javanainen, Phys. Rev. Lett. 57, 3164 (1986).

[7] A. Smerzi, S. Fantoni, S. Giovannazzi, and S. R. Shenoy, Phys. Rev. Lett. , to appear.

[8] S. Raghavan, A. Smerzi, S. Fantoni, and S. R. Shenoy, submitted.

[9] I. Zapata, F. Sols, and A. Leggett, cond-mat/9707143.

[10] R. J. Dodd, K. Burnett, M. Edwards, and C. W. Clark, Phys. Rev. A 56, 586 (1997).

[11] F. Dalfovo and S. Stringari, Phys. Rev. A 53, 2477 (1996); F. Dalfovo et al., Phys. Rev. A 56, 3840 (1997).

[12] D. S. Rokhsar, Phys. Rev. Lett. 79, 1261 (1997).

[13] G. A. Williams and R. E. Packard, Phys. Rev. Lett. 32, 587 (1974); U. Essmann and H. Träuble, Phys. Lett. 24A, 526 (1967).

[14] W. F. Vinen, in Quantum Fluids, edited by D. F. Brewer (North-Holland, Amsterdam, 1966).

[15] W. A. Little and R. D. Parks, Phys. Rev. Lett. 9, 9 (1962).

[16] A. L. Fetter, Phys. Rev. 138 A429 (1965); 138 A709
$(1965)$.

[17] F. Bloch, Phys. Rev. A 7, 2187 (1973); S. J. Putterman, M. Kac, and G. E. Uhlenbeck, Phys. Rev. Lett. 29, 546 (1972); S. J. Putterman, Chap. 8, Superfluid Hydrodynamics (North-Holland, Amsterdam, 1974).

[18] D. S. Rokhsar, cond-mat / 9709212; E. J. Mueller, P. M. Goldbart, and Y. Lyanda-Geller, cond-mat / 9711106.

[19] L. P. Pitaevskii, Sov. Phys. JETP, 13, 451 (1961); E. P. Gross, Nuovo Cimento 20, 454 (1961); J. Math. Phys. 4, 195 (1963).

[20] X. Zhang and J. C. Price, Phys. Rev. B 55, 3128 (1997).

[21] D. S. Jin et al., Phys. Rev. Lett. 77, 420 (1996); L. You et al., Phys. Rev. A 55, R1581 (1997); A. Smerzi and S. Fantoni, Phys. Rev. Lett. 78, 3589 (1997).

[22] K.-P. Marzlin and W. Zhang, cond-mat/9711084.

[23] A. J. Leggett et al., Rev. Mod. Phys. 59, 1 (1987).

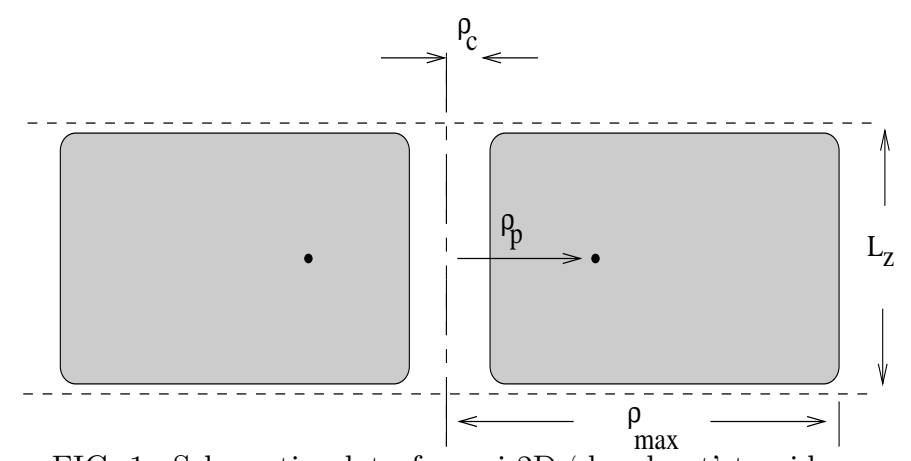

FIG. 1. Schematic plot of quasi-2D 'doughnut' toroid geometry with laser along the $z$ axis. The trap potential rises sharply (dashed boundary) at $L_{z}$. Here $\rho_{c}, \rho_{p}$, and $\rho_{\max }$ are the effective core radius, toroidal axis, and extent of the shaded BEC cloud respectively.

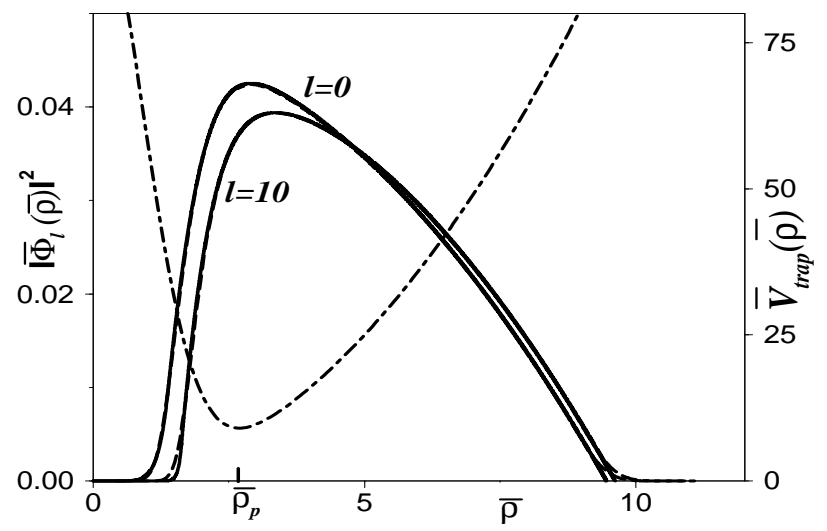

FIG. 2. Trap potential (right vertical axis, dash-dot line) $\bar{V}_{\text {trap }}(\bar{\rho})$ versus cylindrical-coordinate radius, $\bar{\rho}$, (with bars denoting scaling in harmonic trap energy/length). $\bar{\rho}_{p}$ is the effective toroidal trap minimum. Scaled BEC density $\left|\bar{\Phi}_{l}(\bar{\rho})\right|^{2}$ (left vertical axis) versus $\bar{\rho}$ from analytic (solid line) and numerical (dashed line) results. Parameters are as in text. 